Apidologie, 1971, 2 (1), 111-116.

Note TEGHNique

TeChNische MItTEILUNG

\title{
CONSTRUCTION DE DEUX TYPES DE RUCHETTES EXPÉRIMENTALES
}

\section{Konstruktion zweier Typen von Versuchs-Bienenkastchen}

O. Van LAERE

Station de Nématologie et d'Entomologie, (Ministère de l'Agriculture), Wetteren (Belgique)

\section{SUMMARY}

SET UP OF TWO TYPES OF EXPERIMENTAL HIVES

The author describes two types of experimental hives. The first type can accomodate for an indefinite period a small colony of 2,000 to 5,000 bees. It may be fitted with 1,2 or 3 Dadant frames $(43.5 \mathrm{~cm} \times 30 \mathrm{~cm})$, according to the requirements of the experiment.

The second type is designed to accomodate from 50 to 200 bees, with or without queen, for a couple of weeks in supervised environmental conditions (temperature, relative humidity, lighting, food).

\section{RÉSUMÉ}

L'auteur décrit deux types de ruchettes expérimentales.

Le premier type peut abriter pendant un temps illimité une petite colonie de 2000 à 5000 abeilles. Elle peut être pourvue de 1,2 ou 3 cadres Dadant $(43,5 \mathrm{~cm} \times 30 \mathrm{~cm})$, le tout selon les besoins de l'expérience.

Le second type est conçu pour loger de 50 à 200 abeilles, avec ou sans reine, pendant un couple de semaines dans des conditions de milieu (température, humidité relative, éclairage, nourriture) surveillées. 


\section{INTRODUCTION}

A mesure que l'on approfondit certains problèmes de la biologie des abeilles, on se trouve amené à élaborer des méthodes permettant d'élever un groupe d'abeilles ou une colonie entière dans des conditions de milieu soigneusement surveillées.

La ruche classique à 8 ou 12 cadres ne convient pas pour bon nombre d'expériences parce qu'elle est peu accessible et ne se prête donc pas à des inspections fréquentes et n'autorise qu'un contrôle très insuffisant des facteurs de milieu interne.

Le cours de nos recherches nous a conduit à mettre au point les deux types de ruchettes expérimentales que nous décrivons brièvement ci-après.

\section{DESCRIPTION}

\section{1. - Ruchette à trois cadres pour 2000 à 5000 abeilles}

La ruchette est représentée à la fig. 1 . Son plancher et ses parois frontale et dorsale sont en bois ( $3 \mathrm{~cm}$ d'épaisseur). Le plancher présente près du trou de vol un prolongement pourvu d'un rebord de bois couvert de gaze, qui constitue un couloir de sortie long d'environ $20 \mathrm{~cm}$. Cet aménagement permet d'installer la ruchette au laboratoire : l'issue de la ruchette étant placée devant une ouverture pratiquée dans une fenêtre du local, les abeilles peuvent s'envoler et revenir librement. La couverture de gaze du couloir de sortie empêche la pénétration directe d'air extérieur froid dans la ruchette.

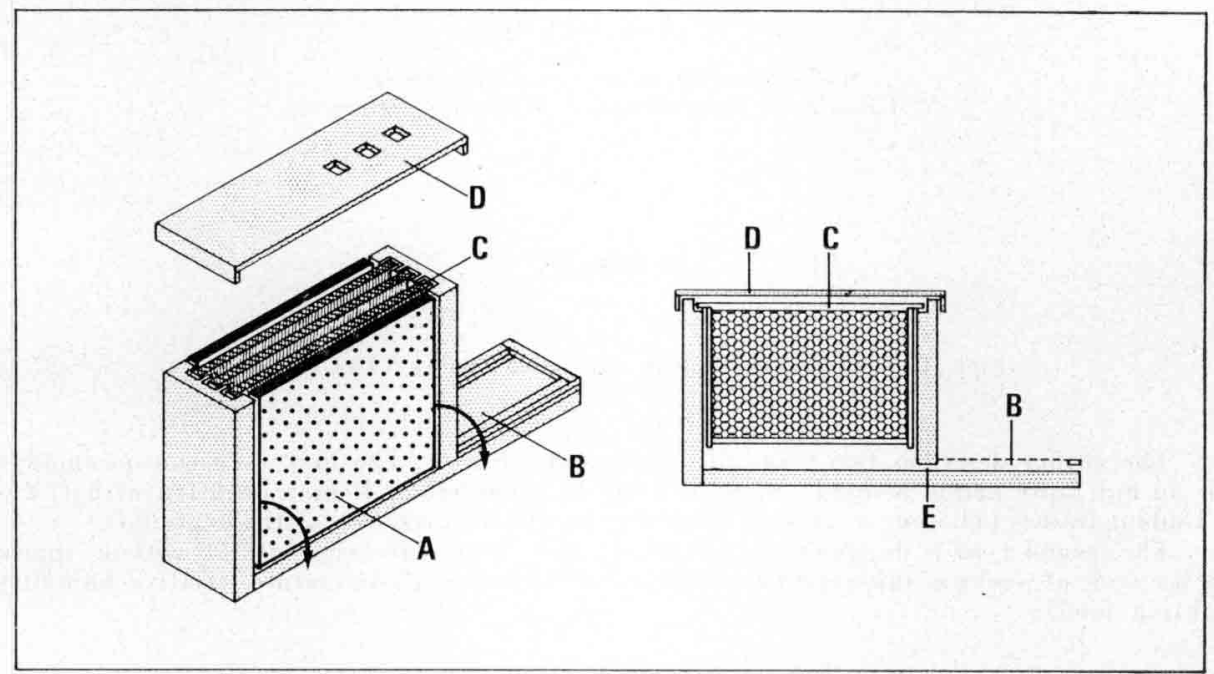

Fic. 1. - Ruchette expérimentale.

A. Paroi latérale de verre double (Thermopane).

B. Couloir de sortie couvert de gaze métallique.

C. Cadre.

D. Couvercle avec ouvertures d'aération, pour le nourrissement, pour le contrôle de la température etc.

E. Issue.

Aвв. 1. - Versuchskästchen.

A. Seitenwand aus Doppelscheiben (Thermopane).

B. mit Drahtgaze abgedeckter Flugkanal.

C. Rähmchen.

D. Deckel mit Öffnungen für Lüftung, Fütterung, Temperaturkontrolle usw,

E. Flugloch. 
Les parois latérales (fig. 1 , A) sont constituées d'un double vitrage (“ Termopane 》). Elles sont simplement posées sur le plancher et glissées entre les parois frontale et dorsale, de sorte qu'on peut facilement les abattre vers l'extérieur (dans le sens indiqué par les flèches) quand l'expérience nécessite l'ouverture de la ruchette. Elles sont maintenues en position verticale par deux petites vis à crochet posées aux coins supérieurs.

Nous avons pu constater que le pouvoir isolant du double panneau de verre est suffisant pour permettre à une petite colonie d'abeilles de se développer par ses propres moyens sur 2 ou 3 cadres pendant la bonne saison.

Un moyen de chauffage d'appoint peut toutefois être souhaitable quand les circonstances deviennent défavorables (absence de miellée, températures nocturnes plus fraîches). Nous avons remplacé à cette fin le panneau de verre par un double vitrage de Plexiglas (polymère de métacrylate de méthyle) constitué de 2 panneaux épais de $4 \mathrm{~mm}$ séparés par un creux de $6,5 \mathrm{~mm}$. Des fils de résistance électrique sont tendus parallèlement, à $3 \mathrm{~cm}$ d'intervalle, dans ce creux : la quantité de chaleur additionnelle peut ainsi être dosée exactement à l'aide d'un transformateur réglable et est uniformément distribuée sur toute la surface des parois latérales. Deux thermomètres placés dans la ruchette permettent de vérifier à tout moment si la température mesurée hors de la grappe d'abeilles ne dépasse pas environ $27^{\circ} \mathrm{C}$ et $20^{\circ} \mathrm{C}$ en hiver.

Si une expérience nécessite des prélèvements d'abeilles à des moments donnés, on peut pratiquer des ouvertures dans les parois latérales, de la manière proposée par Douault (1968) dans une intéressante contribution.

Le couvercle est une simple plaque de bois, plate, avec, à l'avant et à l'arrière, un petit rebord qui enserre les parois frontale et dorsale. Il est pourvu de trois trous d'aération tendus de gaze. Une de ces ouvertures peut, le cas échéant, être réservée pour l'installation d'un nourrisseur.

La ruchette peut facilement être transformée en type à un ou deux cadres par simple enlèvement de deux ou un cadre et rapprochement des parois latérales.

L'espace libre entre la latte supérieure du cadre et le couvercle doit mesurer au moins $1,5 \mathrm{~cm}$ afin de ne pas gêner les allées et venues des abeilles lors du nourrissement.

Un espace libre d'environ $6 \mathrm{~cm}$, ménagé dans le bas de la ruchette, s'est révélé particulièrement utile dans des cas de surpopulation due à un développement exceptionnellement marqué du nid à couvain.

\section{2. - Ruchette fermée pour 50 à 200 abeilles}

De nombreuses recherches nécessitent le maintien des animaux d'essais dans des conditions de milieu sévèrement contrôlées.

La conception de la ruchette que nous avons construite à cette fin s'inspire de la ruchette dite “ de Liebefeld », connue depuis longtemps et de la ruchette décrite par Pain (1966), mais présente un certain nombre de particularités, que nous décrivons ci-dessous (fig. 2).

Les dimensions extérieures (sans les espaces de nourrissement) sont : longueur $12 \mathrm{~cm}$, largeur $6 \mathrm{~cm}$, hauteur $10 \mathrm{~cm}$. Le matériau que nous avons utilisé est le Plexiglas (polymère de métacrylate de méthyle). Ce matériau est à préférer au bois parce qu'il permet un nettoyage approfondi, indispensable quand on procède à des expériences où interviennent des substances odorantes, des phéromones, etc.

La ruchette proprement dite est constituée de deux pièces en $U$ faciles à démonter, de sorte que toutes les surfaces internes sont totalement accessibles pour le nettoyage après l'emploi. Une ou deux bandes élastiques assurent la solidité de l'ensemble.

Une large ouverture, à laquelle s'adapte un couvercle, est pratiquée dans la surface supérieure. Un fragment de rayon est soudé au couvercle.

De larges orifices d'aération sur lesquels un carré de toile métallique est maintenu par 

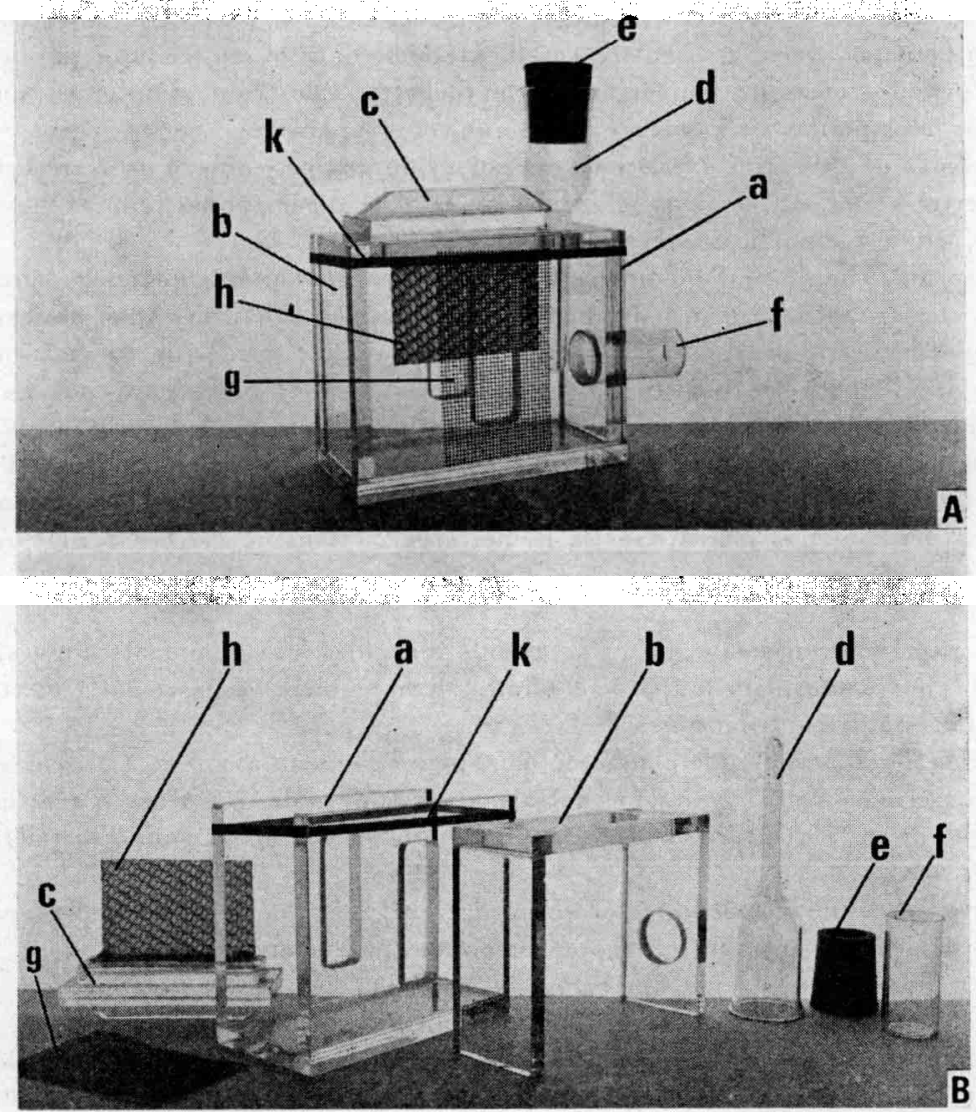

FıG. 2. - Ruchette fermée pour l'élevage de 50 à 200 abeilles en conditions de milieu surveillées.

$$
\text { A. Montée. }
$$

B. Démontée.

$a$ et $b$ : élément en $U$ de la ruchette.

$$
c: \text { couvercle. }
$$

$d$ et $e$ : abreuvoir et bouchon de caoutchouc.

$f$ : tube de nourrissement.

$$
\begin{gathered}
g: \text { gaze. } \\
h \text { : fragment de rayon. } \\
k \text { : bande élastique. }
\end{gathered}
$$

Aвв. 2. - Geschlossenes Bienenkästchen für die Haltung von 50-200 Bienen bei kontrollierten Milieubedingungen.

A. Zusammengebaut.

B. Auseinandergenommen.

$a+b$. U-förmige Elemente des Kästchens.

$$
\text { c. Deckel. }
$$

$d+e$. Trünke u. Gummistopfen.

$f$. Futterröhrchen.

$$
\text { g. Gaze. }
$$

h. Wabenstücke.

h. Gummiband.

des bandes adhésives, sont ménagés dans les deux grandes parois latérales. La toile métallique est enlevée après l'emploi et remplacée par un nouveau carré. Cette solution est meilleure que celle qui consiste à forer de nombreux petits orifices dans la paroi; ces trous s'encollent de résidus de cire et de propolis qui posent de sérieux problèmes de nettoyage, 
Une ouverture ronde de $25 \mathrm{~mm}$ de diamètre est pratiquée dans une des deux petites parois latérales et reçoit un petit tube de verre rempli de candi.

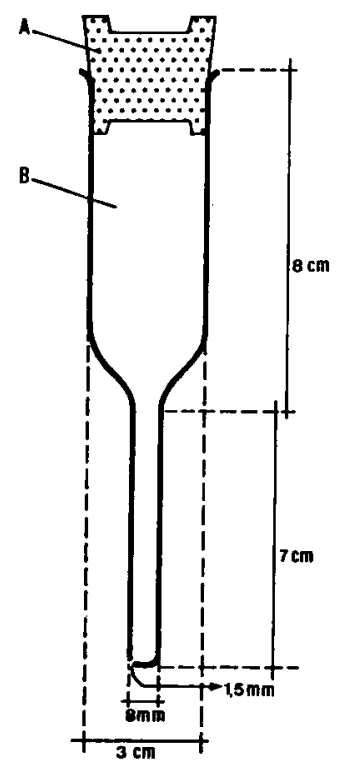

Fig. 3. - Détail de l'abreuvoir.

A. Bouchon de caoutchouc.

$$
\text { B. Tube de verre. }
$$

AвB. 3. - Einzelleile der Tränke.

A. Gummistopfen.

B. Glasröhrchen.

Une ou plusieurs coupelles-mangeoires supplémentaires peuvent être placées sur le plancher de la ruchette, par exemple pour servir du pollen pur quand l'expérience le requiert.

Un petit abreuvoir de verre peut être introduit dans la ruchette par une ouverture de la paroi supérieure (fig. 3).

\section{CONCLUSION}

La ruchette à 3 cadres Dadant s'est révélée particulièrement commode pour les expériences de biologie où les abeilles doivent séjourner dans des conditions aussi proches que possible des conditions naturelles.

Elle peut être installée au laboratoire tout en permettant le libre envol des abeilles et assure ainsi une observation aisée et continuelle des différents phénomènes que l'on se propose d'étudier.

La petite ruchette pour 50 à 200 abeilles permet de garder celles-ci un couple de semaines dans des conditions de milieu constantes et strictement contrôlées. Elle offre un certain nombre d'avantages qui représentent un perfectionnement par rapport à d'autres modèles. 


\section{ZUSAMMENFASSUNG}

Verfasser beschreibt zwei Typen von Versuchs-Bienenkästchen. Beim ersten Typ handelt es sich um ein Dreiwaben-Kästchen für 2 000-3 000 Bienen, dessen Boden, Vorder-und Rückwand aus Holz gefertigt sind. Ein mit Gaze abgedeckter Flugkanal, dessen Ausgang sich vor einer im Fenster eingelassenen Öffnung befindet, erlaubt die Aufstellung des Kästchens im Labor.

Die Seitenwände bestehen aus Plexiglas-Doppelscheiben, die einfach auf dem Boden aufgesetzt sind und so zwischen Vorder-und Rückwand eingeschoben werden, dass sie leicht nach aussen umgekippt werden können, wenn der Versuch die Öffnung des Kästchens erfordert.

Doppelscheiben aus Plexiglas erlauben den Einbau einer Zusatzheizung. Dazu werden die Heizdrähte in Abständen von $3 \mathrm{~cm}$ parallel im Innern der Doppelscheibe gespannt.

Die Umänderung dieses Kästchens in Ein-oder Zweiwaben-Kästchen ist leicht zu bewerkstelligen.

Der zweite Typ ist ein geschlossenes Plexiglas-Kästchen für 50 bis 200 Bienen, dessen Aussenmasse $12 \times 6 \times 10 \mathrm{~cm}$ betragen. Plexiglas ist Holz vorzuziehen weil es sich gründlicher reinigen lässt, was bei Versuchen, in denen mit Duftstoffen, Pheromonen usw. gearbeitet wird, unerlässlich ist.

Die Einzelheiten der Konstruktion sind in Abbildung 2 uns 3 dargestellt.

\section{REFERENCES BIBLIOGRAPHIQUES}

Dounult Ph., 1968. Description et utilisation de deux types de ruchettes expérimentales mono-cadre et bi-cadre. Ann. A beille, 11, 1, 63-67.

PaIN J., 1966. Nouveau modèle de cagettes expérimentales pour le maintien d'abeilles en captivité. Ann. A beille, 9, 1, 71-76. 